\title{
Eine starke Allianz der klinischen Neurodisziplinen
}

\author{
Bassetti, C L ; Merlo, A ; Steinlin, M ; Valavanis, A ; Weder, B
}

\begin{abstract}
Strategische und zukunftsweisende Projekte anstossen - das will die Swiss Federation of Clinical Neuro-Societies. Sie bündelt die Interessen von 13 Gesellschaften und Vereinigungen und versteht sich als Interessenvertretung gegenüber Politik und Gesundheitssystem. Lesen Sie mehr über ihre Aktivitäten zur Hochspezialisierten Medizin, über das Human Brain Project und künftige Kongresse.
\end{abstract}

Posted at the Zurich Open Repository and Archive, University of Zurich ZORA URL: https://doi.org/10.5167/uzh-79525

Journal Article

Published Version

Originally published at:

Bassetti, C L; Merlo, A; Steinlin, M; Valavanis, A; Weder, B (2013). Eine starke Allianz der klinischen Neurodisziplinen. Schweizerische Ärztezeitung (SÄZ), 94(23):870-873. 


\section{Eine starke Allianz der klinischen Neurodisziplinen}

\author{
C. Bassetti, A. Merlo*, \\ M. Steinlin, A. Valavanis, \\ B. Weder
}

SFCNS-Vorstand 2011-2013 (*Vorstandsmitglied bis Mai 2013)

\footnotetext{
1 Bassetti C. Swiss Federation of Clinical Neuro-Societies SFCNS. Schweiz Ärztezeitung. 2010;91(8):296.
}

Korrespondenz: Petra Zalud IMK

Institut für Medizin und

Kommunikation AG

Münsterberg 1

CH-4001 Basel

petra.zalud[at]imk.ch
Gegründet im Jahr 2009 [1] als Zusammenschluss von sechs Gesellschaften ${ }^{* *}$ ) aus den klinischen Neurodisziplinen, bündelt die SFCNS inzwischen die Interessen von 13 Gesellschaften und Interessenvereinigungen, hinter denen insgesamt weit über 1000 Mitglieder stehen:

- Schweizerische Neurologische Gesellschaft $\mathrm{SNG}^{* *}$

- Schweizerische Gesellschaft für Neurochirurgie SGNC**

- Schweizerische Gesellschaft für klinische Neurophysiologie - SGKN**

- Schweizerische Gesellschaft für Neuropädiatrie SGNP**

- Schweizerische Gesellschaft für Neuroradiologie - SGNR**

- Schweizerische Gesellschaft für Neuropathologie - SGNPath**

- Schweizerische Gesellschaft für biologische Psychiatrie - SGBP

- Schweizerische Kopfwehgesellschaft - SKG

- Schweizerische Gesellschaft für Neurorehabilitation - SGNR

- Schweizerische Vereinigung der Neuropsychologinnen und Neuropsychologen - SVNP

- Schweizerische Gesellschaft für Verhaltensneurologie-SGVN

- Schweizerische Hirnschlaggesellschaft - SHG

- Schweizerische Liga gegen Epilepsie - EpilepsieLiga

Die SFCNS bildet eine bedeutende Interessenvertretung gegenüber Politik und Gesundheitssystem und ermöglicht, strategische und zukunftsweisende Projekte anzustossen. Der alle drei Jahre stattfindende Kongress liefert eine Plattform für den Wissensaustausch und ermöglicht die Anbahnung und Vertiefung von interdisziplinären Kooperationen zwischen den klinischen Neurodisziplinen aus dem In- und Ausland. Nach dem erfolgreichen Start 2010 soll nun die Zusammenarbeit mit der über 1000 Mitglieder starken Schweizerischen Gesellschaft für Neurowissenschaften (SSN) an den klinisch relevanten Schnittstellen ausgebaut werden. Für das langfristige Patientenwohl ist ein fruchtbarer Austausch zwischen den grundlegenden, translationellen und klinischen Neurowissenschaften essentiell, um neue diagnostische und therapeutische Verfahren zu entwickeln und anzuwenden.

\section{Der zweite SFCNS-Kongress vom 5.-7. Juni 2013 in Montreux}

Vom 2.-4. Juni 2010 fand der erste SFCNS-Kongress in Basel statt, der von allen Teilnehmern als ein grosser Erfolg gewertet wurde. Sechs Gesellschaften haben die SFCNS 2010 gegründet. Die interdisziplinären Themen zerebrovaskuläre Krankheiten, Diagnose und Behandlung von Epilepsien und Krankheiten des Rückenmarks, der Spinalnerven und der Wirbelsäule standen im Fokus. Der zweite SFCNS-Kongress findet nun unter Beteiligung von 13 Gesellschaften der klinischen Neurodisziplinen vom 5. bis 7. Juni 2013 in Montreux statt. 1000 Teilnehmer werden erwartet. Renommierte Referenten aus dem In- und Ausland sprechen zu den folgenden Themen: Functional Restoration, Neuro-Oncology, Neurocritical Care, Depression, Dementia, Chronic Pain, Neurodevelopement, Psychosis, Stroke, Epilepsy, Neuroimmunology, Advanced Neuroimaging, Spine. Flankiert werden die Vorträge von Satellitensymposien und einer grossen Industrieausstellung. In Montreux kann man sich auch einen Überblick verschaffen über die IV-HSM-Mandate sowie den Stand der Zertifizierungsprozesse im Rahmen einer IV-HSM-Session am 6. Juni 2013. Gastredner und Teilnehmer an der Podiumsdiskussion sind unter anderem der FMH-Präsident Dr. Jürg Schlup, der Tessiner Regierungsrat Paolo Beltraminelli und der Präsident des IV-HSM-Fachorgans Prof. Peter Suter.

Das Programm des SFCNS-Kongresses ist zu finden unter www.sfcns.ch

\section{SFCNS-Mandate zur Hochspezialisierten Medizin (IV-HSM)}

\section{Vorgeschichte der IV-HSM in den} klinischen Neurowissenschaften

Die interkantonale Vereinbarung zur hochspezialisierten Medizin (IV-HSM) stützt sich auf das neue KVG ab und ist ein Projekt der kantonalen Gesundheitsdirektorenkonferenz (GDK), mit dem Ziel, die hochspezialisierte Medizin in der Schweiz zu koordinieren. Ein Fachorgan aus Experten unter Leitung von Prof. Peter Suter erarbeitet Vorschläge zuhanden des HSM-Beschlussorgans der GDK. Hochspezialisierte Medizin umfasst jene Bereiche und Leistungen, welche durch ihre Seltenheit, ihr hohes Innovationspotential, durch einen hohen personellen oder technischen Aufwand oder durch komplexe Behandlungsverfahren gekennzeichnet sind. Für die klinischen Neurowissenschaften haben sich zuletzt drei Themen in diesem Zusammenhang herauskristallisiert: 1. Teile der Schlaganfallmedizin, 2. die stereotaktische Therapie der anormalen/ungewollten Bewegungen sowie die Tiefenhirnstimulation bei 
Morbus Parkinson, und 3. die chirurgische Behandlung der refraktären Epilepsie beim Erwachsenen.

Die Beschlüsse der GDK haben Verordnungscharakter und entziehen sich damit einer direktdemokratischen Kontrolle, können aber beim Bundesverwaltungsgericht angefochten werden. Neben den Organtransplantationen war die Schweizerische Gesellschaft für Neurochirurgie (SGNC) früh in das HSM-Projekt involviert [2]. Die Vernehmlassungszeiten waren allerdings sehr kurz bemessen und behinderten die tiefere Auseinandersetzung mit der komplexen Thematik. Umstritten bleibt unter anderem die Aussagekraft von Fallzahlen [3]. Fallzahlen sollten nicht als absolute Grössen, sondern als Richtschnur im Verbund mit anderen Qualitätsindikatoren dienen. Ein weiterer Kritikpunkt ist die kurze Zertifikat-Laufzeit von 3 Jahren. Einige Experten und Gesellschaften plädieren für eine Zertifikat-Laufdauer von 7 Jahren. Andererseits könnte die vorgesehene Aufstellung von Fall-Registern zu einem innovativen Instrument werden, den fachlichen Austausch innerhalb der interdisziplinären Neuro-HSM zu stimulieren und zu intensivieren.

2 Bundesblatt, Nr. 25, 21. Juni 2011, S. 4692-8.

3 Landolt $\mathrm{H}$, Merlo A. Hochspezialisierte Medizin und die Neurochirurgie. Schweiz Med Forum. 2011;11(3):37-39.

4 Merlo A, Bernays R, Hausmann O, Landolt H, Reinert M, Schaller K. Nutzen der Zentralisierung hochspezialisierter Leistungen in Frage gestellt. Schweiz Ärztezeitung. 2012;93(31/32):1138-40.

5 Bassetti C, Arnold M, von Below G. Zertifizierung von Stroke Centers und Stroke Units in der Schweiz. Schweiz Ärztezeitung 2012;93(19):706.

6 Lyrer P, Michel P, Arnold M, Hungerbühler H, Gralla J, Humm A, et al. Stroke Units und Stroke Centers in der Schweiz: Richtlinien und Anforderungsprofil. Schweiz Med Forum. 2012;12(47):918-922.

7 Gostynski M, Engelter S, Papa S, Ajdacic-Gross V, Gutzwiller F, Lyrer P. Incidence of first-ever ischemic stroke in the Canton Basle-City, Switzerland: a populationbased study 2002/2003. J Neurol. 2006;253(1):86-91.

8 Meyer K, Simmet A, Arnold M, Mattle H, Nedeltchev K. Stroke events and case fatalities in Switzerland based on hospital statistics and cause of death statistics. Swiss Med Wkly. 2009; 139(5-6):65-9.
Zertifizierung von Stroke Centers und Stroke Units In seinem Entscheid vom 20. Mai 2011 betreffend die hochspezialisierte Behandlung von Hirnschlagpatientinnen und -patienten hat das IV-HSM-Beschlussorgan der GDK beschlossen, dass schweizweit acht Stroke Centers (Schlaganfallzentren) den Teil der hochspezialisierten klinischen Versorgung, die Aus- und Weiterbildung sowie die klinische Forschung im Bereich Stroke massgeblich wahrnehmen sollen [4]. Die Zertifizierung der hochspezialisierten Stroke Centers wurde als Voraussetzung für diese Aufgabe formuliert, das Zertifizierungsmandat wurde der Swiss Federation of Clinical Neuro Societies (SFCNS) als dem Dachverband der klinischen Neurowissenschaften übertragen [5].

Die SFCNS-Stroke-Zertifizierungskommission erarbeitete auf der Grundlage der von der Schweizerischen Hirnschlaggesellschaft (SHG) entwickelten Richtlinien [6] sowie in Anlehnung an die Richtlinien der European Stroke Organisation (ESO) das Anforderungsprofil für Stroke Centers und Stroke Units. Im Leistungsspektrum der Stroke Units sind die als hochspezialisiert definierten Behandlungsverfahren ausgeschlossen. Die Zertifizierungskriterien und -verfahren wurden in Zusammenarbeit mit der Stiftung sanaCERT ausgearbeitet.

Jährlich erleiden rund 15000 Personen in der Schweiz einen Hirnschlag und schätzungsweise 5000 eine Transitorische Ischämische Attacke (TIA) [7,8]. Um eine flächendeckende, hochspezialisierte Versorgung zu gewährleisten, sollen die acht Stroke Centers in regionalen Netzwerken mit Stroke Units zusammenarbeiten.

\section{Zur Definition}

Eine Stroke Unit (SU) ist eine Behandlungseinheit eines Spitals, die für Hirnschlagpatienten konzipiert ist. Die Behandlung in einer SU ist für alle Schweregrade und alle Altersgruppen von Patienten mit Hirnschlag wirksam. Sie verfügt über monitorisierte und nicht-monitorisierte Behandlungsplätze.

Ein Stroke Center (SC) umfasst eine SU und erweitert das Konzept der SU um spezifische strukturelle, neuroradiologische und neurochirurgische Leistungen.

Zahlreiche Kliniken hatten 2012 bereits ihr prinzipielles Interesse an einer Zertifizierung als Stroke Center oder Stroke Unit der GDK mitgeteilt. Stroke Centers und Stroke Units können sich über das SFCNS-Zertifizierungsbüro für eine Zertifizierung formell anmelden. Alle erforderlichen Unterlagen sind auf der Internetseite der SFCNS zu finden oder auf Anfrage bei der SFCNS-Geschäftsstelle zu erhalten.

\section{Zertifizierungsablauf}

Nach einer Vorprüfung durch die SFCNS-Projektadministration regelt ein Vertrag zur Zertifizierung die weiteren Abläufe. Das Spital stellt dem Auditorenteam gemäss einer Dokumentenliste umfassendes Material zum Nachweis der Erfüllung der erforderlichen Qualitätskriterien zur Verfügung. Diese betreffen Personal, Infrastruktur, Prozesse, Forschung, Weiterbildung und Fallzahlen. Sogenannte MussKriterien, 12 bei Stroke Centers und 8 bei Stroke Units, müssen erfüllt werden; ohne diese ist eine Zertifizierung nicht möglich. Weitere Kriterien gehen mit einer Punkteskala von 1-3 in die Bewertung ein. Zwei Auditoren (medizinische Fachpersonen mit einer erweiterten, dokumentierten Kenntnis und mehrjähriger Führungserfahrung im Bereich der Hirnschlagversorgung) und ein leitendender Auditor der sanaCERT Suisse bewerten die eingereichten Unterlagen. Ergänzende Informationen werden im Rahmen eines ganztägigen Audits vor Ort eingeholt. Hier werden Stichproben aus Falldokumentationen gezogen sowie Interviews mit der SC/SU-Leitung und Mitgliedern des Stroke Team geführt. In einem Abschlussbericht fassen die Auditoren ihre Beurteilung zusammen. Allfällige Empfehlungen zu Verbesserungen oder Weiterentwicklungen können gegeben werden. Die Zertifizierung wird nach positivem Entscheid durch die SFCNSHirnschlagkommission 6-9 Monate nach Anmeldung beurkundet und ist auf 3 Jahre befristet. Als erstes Stroke Center der Schweiz durchlief das Kantonsspital St. Gallen (KSSG) erfolgreich den Zertifizierungsprozess.

«Die Zertifizierung der Stroke Centers und Stroke Units ist ein Meilenstein auf dem Weg zur Qualitätssicherung und Exzellenz der medizinischen Versorgung von Schlaganfällen in der Schweiz», so der SFCNS-Präsident Prof. Claudio Bassetti. «In einem einjährigen Prozess und unter Einbindung von Fachkollegen aus allen relevanten Disziplinen konnten die Zertifizierungskriterien und -prozesse ausgearbeitet werden.» 
Die SFCNS-Zertifizierungskommission Stroke hat mit grossem Engagement den Grundstein zur Umsetzung einer hoheitlichen Aufgabe gelegt. Sie setzt sich wie folgt zusammen:

- Prof. Dr. Marcel Arnold (Präsident), Inselspital Bern;

- Prof. Dr. Stefan Engelter, Universitätsspital Basel;

- PD Dr. Patrik Michel, CHUV Lausanne;

- Prof. Dr. Karl Schaller, HUG, Genf;

- Prof. Dr. Anton Valavanis, USZ Zürich,

In einem nächsten Schritt soll ein nationales Register die Qualitätssicherung flankieren.

\section{Zertifizierung von} neurochirurgischen Behandlungsverfahren Wie einleitend dargelegt, wurde die hochspezialisierte Medizin in der Schweiz in der Neurochirurgie als eine der ersten Fachgesellschaften strukturiert. Während die Mehrzahl der neurochirurgischen HSM-Themen eigenständig abgehandelt werden, sind die interdisziplinären Bereiche Tiefenhirnstimulation und Epilepsiechirurgie für den Zertifizierungsprozess und insbesondere für die Registerführung an die SFCNS delegiert worden.

Gemäss Entscheid vom 20. Mai 2011 zur Planung der hochspezialisierten Medizin (HSM) im Bereich der stereotaktischen Chirurgie der anormalen/ungewollten Bewegungen und der tiefen Hirnstimulation, Deep Brain Stimulation, beim Morbus Parkinson hat das IV-HSM-Beschlussorgan der GDK festgelegt, die Behandlungen in vier dafür spezialisierten Zentren zu konzentrieren[9].

Die chirurgische Behandlung der refraktären Epilepsie soll sich in drei dafür spezialisierten Zentren konzentrieren [10].

Die Konzentration ergibt sich einerseits aus den begrenzten Fallzahlen in der Schweiz (ca. 100 Patienten p.a.), der notwendigen Expertise eines interdisziplinären Behandlungsteams sowie der aufwendigen Nachbetreuung und der spezifischen apparativen Ausstattung. Die Konzentration soll da erfolgen, wo schon ein Aufbau der Infrastruktur, beträchtliches Wissen, Erfahrung und Kompetenz über die letzten Jahre gesammelt wurden.

Der SFCNS wurden auch diese beiden Zertifizierungsmandate übertragen. In zwei SFCNS-Kommissionen werden unter besonderer Mitwirkung der Schweizerischen Gesellschaft für Neurochirurgie (SGNC) Qualitätsrichtlinien als Basis für Zertifizierungskriterien erarbeitet; sie betreffen Personal, Infrastruktur, Prozesse, Forschung, Weiterbildung und Fallzahlen. Die Zertifizierungsprozesse folgen den SIWF-Vorgaben. Die Zertifizierung der Zentren soll die Exzellenz der medizinischen Versorgung in der Schweiz auch im internationalen Vergleich sichern.

9 Bundesblatt, Nr. 25, 21. Juni 2011, S. 4675-8.

10 Bundesblatt, Nr. 25, 21. Juni 2011, S. 4196-9.

11 Pressemitteilung Human Brain Project, Jan. 2013.

\section{SFCNS-Zertifizierungskommission DBS:}

- PD Dr. C. Pollo, Inselspital/Bern (Präsident)

- PD Dr. C. Baumann, USZ/Zürich

- Prof. F. Vingerhoets, CHUV/Lausanne

\author{
SFCNS-Zertifizierungskommission Epilepsie- \\ chirurgie: \\ - Prof. L. Mariani, Unispital/Basel (Präsident) \\ - Prof. T. Grunwald, USZ/Zürich \\ - Prof. M. Seeck, HUG/Genf
}

Die Zertifizierungen im Bereich Neurochirurgie sollen ab Sommer 2013 starten. Nähere Informationen unter www.SFCNS.ch

\section{Human Brain Project: Präsentation am SFCNS-Kongress}

Prof. Henry Markram vom Brain Mind Institute der EPFL wird das Europäische Flaggschiff Human Brain Project vorstellen. Mit dem Human Brain Project soll das gesamte bestehende Wissen über das menschliche Gehirn zusammengefasst und dieses Stück für Stück mit supercomputerbasierten Modellen und Simulationen rekonstruiert werden. Diese Modelle bieten die Chance, neue Erkenntnisse über das menschliche Gehirn und seine Erkrankungen zu gewinnen sowie völlig neue Computer- und Robotertechnologien zu entwickeln. Das Projekt wird hierzu über die nächsten 10 Jahre mehr als 80 Europäische und internationale Forschungsinstitute vernetzen. Die Kosten des Projektes werden auf 1,19 Mrd. geschätzt [11].

Die Schweiz spielt eine tragende Rolle beim Human Brain Project. Henry Markram und sein Team von der EPFL werden das Projekt koordinieren und die Verantwortung für die Entwicklung und den Betrieb der Gehirnsimulationsplattform des Projekts tragen. Richard Frackowiak (CHUV) und sein Team sind für die Medizininformatikplattform des Projekts zuständig, und das Swiss Supercomputing Centre in Lugano wird wesentliche Hochleistungsrechnerkapazitäten zur Verfügung stellen. Viele weitere Schweizer Gruppen leisten ebenfalls Beiträge zum Projekt.

\section{Swiss Brain Council SBC:}

Gründungsveranstaltung am SFCNS-Kongress Im Rahmen des Kongresses startet am 6. Juni die Initiative Swiss Brain Council. SBC setzt die Ziele des European Brain Council auf nationaler Ebene um. Im Besonderen geht es um gemeinsames Lobbying für Hirnforschung und für eine verbesserte Lebensqualität von Betroffenen mit Hirnerkrankungen. Das SBC wird konkret Forschende, Kliniker aus dem Neurobereich und Betroffene in Projekten zusammenführen und dadurch die Praxis- und Patientennähe in der Forschung fördern. Zahlreiche Informationsveranstaltungen zu relevanten neurowissenschaftlichen Themen werden im «Year of the Brain 2014» stattfinden.

\section{SFCNS-Kongress in Basel 2016}

Der dritte SFCNS-Kongress findet im September 2016 als gemeinsamer Kongress mit der Schweizerischen Gesellschaft für Neurowissenschaften SSN in Basel statt. 